\title{
Commission 29: Stellar Spectra
}

\author{
PRESIDENT: Christopher Sneden \\ VICE-PRESIDENT: M. Parthasarathy \\ ORGANIZING COMMITTEE: Fiorella Castelli, Katia Cunha, \\ Philippe Eenens, Eileen Friel, Raffaele Gratton, Ivan Hubeny, \\ John D. Landstreet, Gautier Mathys, Nikolai Piskunov, \\ Francesca Primas, Masahide Takada-Hidai, and Werner Weiss
}

PRESIDENT: Christopher Sneden (University of Texas at Austin; metal-poor stars, Galactic chemical evolution, globular clusters, atomic data)

VICE-PRESIDENT: M. Parthasarathy (Indian Institute of Astrophysics; asymptotic giant branch stars, circumstellar dust; stellar chemical compositions

\section{ORGANIZING COMMITTEE:}

Fiorella Castelli (Istituto di Astrofisica Spaziale e Fisica Cosmica, CNR; stellar atmospheres, atomic data, horizontal branch stars)

Katia Cunha (Observatório Nacional/MCT and NOAO; chemical compositions of hot stars; star clusters; Magellanic Clouds)

Philippe Eenens (Univ. de Guanajuato; massive stars, stellar winds, Wolf-Rayet stars)

Eileen Friel (U.S. National Science Foundation; photometry and spectroscopy of Galactic star clusters, chemical compositions)

Raffaele Gratton (Osservatorio Astronomico di Padova; globular star clusters, stellar abundances, cluster variable stars, Galactic chemical evolution)

Ivan Hubeny (NOAO; stellar atmospheres, stellar line formation, hot stars, white dwarf stars)

John D. Landstreet (The University of Western Ontario; peculiar A stars, stellar magnetic fields, spectropolarimetry, variable stars)

Gautier Mathys (European Southern Observatory; chemically peculiar stars, magnetic fields, A-star abundances and pulsations)

Nikolai Piskunov (Uppsala University; Doppler imaging, starspots, magnetic fields, peculiar A stars, stellar atmospheres, atomic data)

Francesca Primas (European Southern Observatory; stellar abundances, very metal-poor stars, light elements in the early Galaxy)

Masahide Takada-Hidai (Tokai University; stellar abundances, chemically peculiar stars, magnetic fields)

Werner Weiss (University of Vienna; stellar model atmospheres; extra-solar planets, A-peculiar stars)

\section{Introduction}

Participants in Commission 29 study various aspects of stellar spectra and the information that can be extracted from spectra. The list of fields of interest of the Organizing Committee members suggest some major current research topics in this area are stellar chemical compositions and surface/envelope phenomena. Some of the topics of this commission have overlap with other commissions, such as Commission 14 (Atomic and Molecular Data), 26 (Double and Multiple Stars), 27 (Variable Stars), 30 (Radial 
Velocities), 36 (Theory of Stellar Atmospheres), and 37 (Star Clusters and Associations). Many Commission 29 members are also members of these other commissions.

Commission 29 has endorsed several IAU Symposia recently, and our members have been active in organizing and participating in these meetings. In the next section very brief descriptions will be given of these Symposia.

Note that other proposed meetings were favorably considered by the commission, but ultimately were not chosen for IAU Sponsorship. Here is one prominent example. The meeting on Stellar Pulsations and Evolution, with SOC Chairs G. Bono and A. R. Walker, was originally proposed as an IAU Symposium. This commission viewed it favorably but it was not ultimately selected for IAU sponsorship. Happily, the meeting found other funding sources, and took place June 19-24, 2005. Other official IAU Symposia and Colloquia were endorsed by the related commissions noted above.

\section{Recent and Approved IAU Meetings Endorsed by Commission 29}

Symposium 228, From Lithium to Uranium: Elemental Tracers of Early Cosmic Evolution:, May 23-27, 2005, in Paris, France, dedicated to the careers of M. and F. Spite. SOC Chair: R. Cayrel; LOC Chair: V. Hill. Topics included: Lithium in the early Galaxy, extremely metal-poor stars, supernova nucleosynthesis, chemical compositions of globular clusters, observation and theory of neutron-capture element production, abundances in local group and high redshift galaxies.

Symposium 224, The A-Star Puzzle: July 8-13, 2004, in Poprad, Slovakia, dedicated to the memory of V. L. Khokhlova and Horace W. Babcock. SOC Chairs: W. W. Weiss and J. Zverko. LOC Chair: J. Ziznovský. Topics included: observation and theory of normal and chemically peculiar stars; stellar evolution and rotation; binarity; magnetic fields, diffusion and convection; evolved A-stars; pulsating variables.

Symposium 239, Convection in Astrophysics: August 21-25, 2006, in Prague, Czech Republic. SOC Chairs: F. Kupka and I. Roxburgh. Topics will include: observations and modeling of convection and radiative transfer; convection in planets and brown dwarfs; the role of convective mixing in stellar evolution; rotation, oscillations and mass loss; MHD and dynamos.

Christopher Sneden President of the Commission 